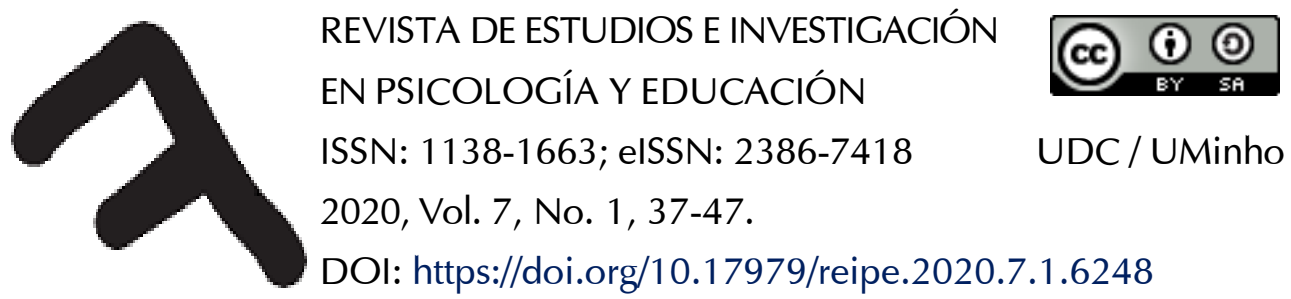

\title{
Aprendizaje de palabras en euskera, altas y bajas en imagen, mediante la mnemotecnia de la palabra clave
}

\section{Learning high and low image vividness words in Euskera using keyword mnemonics}

\author{
Aitziber Goñi-Artola (D)*, María Ángeles González (iD*, Alfredo Campos (iD** \\ *Universidade da Coruña, **Universidad de Santiago de Compostela
}

\begin{abstract}
Resumen
La mnemotecnia de la palaba clave se utiliza para el aprendizaje de una lengua extranjera. Se ha comprobado su eficacia en muchas lenguas en todo el mundo. Este estudio tenía como objetivo analizar la eficacia de la mnemotecnia de la palabra clave en el aprendizaje del idioma euskera, utilizando listas largas de palabras, altas y bajas en imagen. Se ha utilizado un grupo de 424 estudiantes (226 mujeres y 198 hombres) de la Enseñanza Secundaria Obligatoria para que aprendiesen la traducción española de una lista de 28 palabras del euskera ( 14 altas en imagen y 14 bajas en imagen). Fueron administrados el Gordon Test of Visual Imagery Control y el Vividness of Visual Imagery Questionnaire. Se ha comprobado que la mnemotecnia de la palabra clave, cuando la palabra clave es elegida por los compañeros de los participantes, tuvo un mayor recuerdo de la traducción de palabras del euskera al español, que el grupo que utilizó el método de repetición y los grupos que utilizaron otros métodos de selección de la palabra clave. Se discuten los resultados y se proponen nuevas líneas de investigación.
\end{abstract}

Palabras clave: mnemotecnia; palabra clave; idiomas; aprendizaje; recuerdo

\begin{abstract}
Keyword mnemonics has been shown to be an effective method of learning foreign vocabulary in a variety of languages from around the world. This study examined the effectiveness of keyword mnemonics in the learning of Basque using long lists of high and low image vividness words. A sample of 424 (226 female and 198 male) compulsory secondary education students were provided with a list of 28 Basque words (14 high image vividness and 14 low image vividness) and their Spanish translation, and then surveyed using the Gordon Test of Visual Imagery Control and the Vividness of Visual Imagery Questionnaire. The results revealed that when keyword words were chosen by the participants' classmates, recall of the Spanish translation of the Basque words was higher in the keyword mnemonics group than in the groups that used repetition or other methods for selecting keywords. The study ends with a discussion of the results and proposals for new lines of research.
\end{abstract}

Keywords: mnemonics; keyword; languages; learning; recall

Aitziber Goñi-Artola Đ0000-0001-9109-2315 y María Ángeles González D0000-0001-9820-3268: Departamento de Psicología,

Universidade da Coruña. Campus de Elviña s/n, 15071 A Coruña, España.

Alfredo Campos [D0000-0002-4191-4648: Departamento de Psicología Básica, Universidad de Santiago de Compostela. Rúa Xosé María Suárez Núñez s/n, Campus sur. 15782 Santiago de Compostela, España.

Correspondencia relativa a este artículo: Alfredo Campos alfredo.campos@usc.es 
La mnemotecnia de la palabra clave se utiliza, fundamentalmente, para el aprendizaje de idiomas. Fue creada por Atkinson (1975) para el aprendizaje del idioma ruso por parte de sujetos de habla inglesa. Desde su creación se ha extendido su uso por todo el mundo. La regla mnemotécnica consta de dos partes, en primer lugar, se elabora una palabra clave, lo más concreta posible, que suene lo más parecida posible a la palabra extranjera que se desea aprender. En segundo lugar, se crea una imagen mental que una el significado de la palabra clave con el significado de la palabra que se quiere aprender (Atkinson, 1975; Campos, Amor, \& González, 2002; Campos, Camino, \& Pérez-Fabello, 2011; González, Amor, \& Campos, 2003). Las investigaciones indican que la forma en que la mnemotecnia resulta más efectiva es mediante la aplicación individual (Levin, Pressley, McCormick, Miller, \& Shriberg, 1979), cuando es utilizada por niños (Levin, McCormick, Miller, Berry, \& Pressley, 1982), y es menos eficaz con adultos (Troutt-Ervin, 1990).

Un aspecto importante de la mnemotecnia de la palabra clave, y que va a influir mucho en su eficacia, es la forma de elaborar la palabra clave. La forma habitual de utilizar la palabra clave es facilitándola el experimentador a los participantes en el momento del aprendizaje, o diciendo a los participantes que la vayan generando en el momento del estudio (ver González et al., 2003, para una revisión). Un método y otro tienen ventajas e inconvenientes. El hecho de que facilite la palabra el experimentador tiene la ventaja de que el participante sólo tiene que preocuparse del aprendizaje, y no tiene que perder el tiempo intentando buscar las palabras clave, sobre todo cuando el tiempo está cronometrado y es escaso, pero, también tiene el inconveniente de que puede ser que las palabras que seleccionó el experimentador no sean significativas para los individuos que las tienen que aprender. El hecho de que sea el participante el que genere las palabras clave cuando está aprendiendo los significados de las palabras extranjeras tiene la ventaja de que son las palabras clave significativas para los individuos, pero, este método tiene el inconveniente de que, en muchas ocasiones el individuo gasta todo el tiempo en la generación de la palabra clave sin darle tiempo al estudio de los significados de las palabras extranjeras (Campos, González, \& Amor, 2004; González et al., 2003).

Campos y colaboradores (Campos, Amor, \& González, 2002, 2004a, 2004b; Campos, González, \& Amor, 2004) idearon un método de generar las palabras clave que tiene las ventajas de los dos métodos anteriores, $\mathrm{y}$ no tiene los inconvenientes. El método consiste en que las palabras clave, en vez de generarlas el experimentador, las generen compañeros de los participantes, que tengan las mismas características socioeducativas que los participantes. De este modo, las palabras que generen, sin limitación de tiempo, se parecerán mucho a las palabras clave que generarían los participantes. A la hora del aprendizaje, estas palabras, generadas por los compañeros, serán las que presente el experimentador en el momento del aprendizaje (Campos, Amor et al., 2002). Con este nuevo método de selección de la palabra clave, los resultados obtenidos en el aprendizaje de una lengua fueron superiores a otros métodos, tanto con jóvenes (Campos, Amor et al., 2002, 2004a, 2004b; Campos, González et al., 2004), como con personas adultas (Campos \& Ameijide, 2014; Campos, Pérez-Fabello, \& Camino, 2010).

El método de la palabra clave se ha utilizado con éxito en el aprendizaje de muchos idiomas, como el ruso (Atkinson, 1975; Atkinson \& Raugh, 1975), el latín (Campos, González et al., 2004; Shapiro \& Waters, 2005), español (Hall, Wilson, \& Patterson, 1981; Pressley, Samuel, Hershey, Bishop, \& Dickinson, 1981; Raugh \& Atkinson, 1975), el francés (Merry, 1980), inglés (Baleghizadeh \& Ashoori, 2010; Davoudi \& Yousefi, 2016), italiano (Hogben \& Lawson, 1994; Lawson \& Hogben, 1998), y el gallego (Campos, Rodríguez-Pinal, \& Pérez-Fabello, 2013, 2014).

Si bien existen muchas investigaciones sobre la eficacia de la mnemotecnia de la palabra clave en los diversos idiomas del mundo (ver González et al., 2003, para una revisión), sólo existen dos trabajos que relacionan la mnemotecnia de la palabra clave y el euskera. El primero (González, Goñi-Artola, \& Campos, 2019) analiza el efecto de la concordancia en la generación de la palabra clave entre los compañeros de los participantes en el aprendizaje del euskera mediante la mnemotecnia de la palabra clave. Se encontró que las palabras clave, altas en concordancia, producían un mayor aprendizaje del euskera que las palabras clave bajas en concordancia, tanto inmediatamente, como al cabo de una semana.

En el segundo artículo (González, Goñi-Artola, \& Campos, 2020) se presentó una lista corta, 14 palabras, 
para que diferentes grupos de estudiantes de Enseñanza Secundaria Obligatoria las aprendiesen mediante la mnemotecnia de la palabra clave (palabras clave seleccionadas por los experimentadores, palabras clave generadas por los propios participantes en el momento de aprendizaje, y palabras clave seleccionadas por compañeros de los participantes) y mediante el método de repetición. Se encontró que el grupo de participantes que utilizó el método de la palabra clave seleccionadas por los compañeros tuvo un aprendizaje superior a los otros grupos. No se encontraron diferencias significativas en la eficacia del control de imagen (la facilidad que tiene el individuo para ver la misma imagen en distintas posiciones), pero sí en viveza de imagen (habilidad del individuo para imaginarse las cosas con tanta claridad como si las estuviese percibiendo). Las personas altas en viveza de imagen tuvieron un mayor aprendizaje del significado de palabras en euskera que los individuos bajos en viveza de imagen.

Una de las principales variables que influyen en la mnemotecnia de la palabra clave es el control de imagen que tienen los individuos. Campos et al. $(2013,2014)$ utilizaron la mnemotecnia de la palabra clave con alumnos de Educación Secundaria Obligatoria, y también les aplicaron el Gordon Test of Visual Imagery Control (Richardson, 1969). Los resultados de su investigación indicaron que los individuos que eran altos en control de imagen tuvieron un mejor recuerdo de la traducción española de las palabras en gallego que los participantes que tenían bajo control de imagen.

Además del control de imagen, otra variable que influye en el rendimiento cuando los participantes utilizan alguna regla mnemotécnica que utiliza las imágenes, es la habilidad para formar imágenes mentales vivas (Campos, González et al., 2004; Denis, 1979; Paivio, 1971; Richardson, 1994). Diferentes estudios relacionan la habilidad de formar imágenes vivas y la memoria, sobre todo si los ítems que se tienen que aprender son palabras concretas con alto valor de imagen (ver Ernest, 1991, y Richardson, 1994, para una revisión). Campos, González et al. (2004) encontraron que la habilidad de formar imágenes mentales vivas influía significativamente en el recuerdo. Campos y Pérez (1997) también encontraron que la habilidad de formar imágenes influía significativamente en el recuerdo inmediato, tanto de listas cortas (16 palabras) como de listas largas (32 palabras). Campos, Amor et al. (2002) utilizaron una lista de 30 palabras latinas para ser aprendidas por individuos de habla hispana mediante la mnemotecnia de la palabra clave, y encontraron que los individuos altos en habilidad para formar imágenes mentales tuvieron un mejor recuerdo inmediato y a largo plazo, que los individuos que tenían baja habilidad para formar imágenes mentales. Por su parte, Wyra, Lawson, y Hungi (2007) utilizaron una lista de 22 palabras para aprenderlas mediante la mnemotecnia de la palabra clave, y también encontraron diferencias en el recuerdo entre los individuos altos y bajos en habilidad para formar imágenes vivas.

A la hora del aprendizaje mediante imágenes, una variable importante es el nivel que tienen las palabras de suscitar una imagen. Las palabras concretas, en general, suscitan una mayor imagen que las palabras abstractas (Sadoski, 2005). Lawson y Hogben (1998) observaron que las palabras altas en imagen se recordaban mejor que las bajas en imagen cuando se utilizaba la mnemotecnia de la palabra clave. A la misma conclusión llegaron las investigaciones llevadas a cabo por Beaton, Gruneberg, Hyde, Shufflebottom, y Sykes (2005), Shapiro y Waters (2005), Campos, Camino y Pérez-Fabello (2010), y Wei (2015), todos ellos utilizando la mnemotecnia de la palabra clave.

Los resultados que se obtienen mediante la mnemotecnia de la palabra clave se interpretan (Campos et al, 2014) a la luz de la teoría de la Memoria Episódica de Tulving (2002), que sostiene que el material almacenado en la memoria episódica tiene un buen recuerdo inmediato, pero se olvida más fácilmente que otros tipos de memoria. También se interpretan a la luz de la Teoría de la Codificación Dual de Paivio (1971), que sostiene que el material aprendido mediante imágenes, como la mnemotecnia de la palabra clave, se codifica mediante imágenes y lenguaje, en cambio, cuando el material se aprende sólo de forma verbal, se codifica sólo mediante un sistema. Por supuesto, esto tiene el inconveniente del fácil olvido.

En este trabajo se ha tratado de analizar si la mnemotecnia de la palabra clave, el control de imagen y la viveza de imagen influyen en el recuerdo inmediato de listas largas de palabras, altas en imagen, bajas en imagen, y en el total de palabras del euskera. De acuerdo con ello, la hipótesis que se ha planteado es que la 
estrategia de aprendizaje (método de la palabra clave suscitada por los compañeros, por los experimentadores, los participantes, y el método de repetición), el control de imagen (participantes altos y bajos en control de imagen), y viveza de imagen (participantes altos y bajos en viveza de imagen) influyen en el recuerdo inmediato de palabras del euskera, altas en imagen, bajas en imagen y total de palabras. Las variables independientes fueron la estrategia de aprendizaje, el control de imagen, y la viveza de imagen. Como variable dependiente se utilizó el recuerdo del significado de palabras del euskera, altas en imagen, bajas en imagen, y total de palabras.

La novedad fundamental de este trabajo reside en analizar la influencia de la mnemotecnia de la palabra clave en el euskera, utilizando listas largas de palabras, altas y bajas en imagen. De hecho, son escasos los estudios que analizan la influencia de la mnemotecnia de la palabra clave en el aprendizaje del euskera, como lengua extranjera.

\section{Método}

\section{Participantes}

En esta investigación participaron 424 estudiantes de los cursos $3^{\circ}$ y $4^{\circ}$ de Enseñanza Secundaria Obligatoria (ESO), de colegios públicos de la ciudad de A Coruña (España). El grupo estaba formado por 198 varones $(46.7 \%)$ y 226 mujeres, con un rango de edad de entre 14 y 18 años $(M=15$ años, $D T=0.87)$.

\section{Instrumentos}

En esta investigación se empleó una lista de 28 palabras en euskera, de las que 14 eran altas en imagen, y otras 14 eran bajas en imagen. También se utilizaron 28 palabras clave y la traducción española de las palabras en euskera. Además, se utilizaron 28 frases interactivas en las que relacionaban las palabras clave y el significado español de las palabras en euskera.

Se utilizó la versión española (Pérez-Fabello \& Campos, 2004) del Gordon Test of Visual Imagery Control (Richardson, 1969). El cuestionario consta de 12 ítems, y mide el control de imagen de los participantes. Cada ítem del cuestionario se puntúa del 0 al 2, siendo un 0 cuando la respuesta es un "no", un 1 cuando la respuesta es "inseguro", y cuando la respuesta es un "sí" se puntúa con un 2.

También hemos se ha utilizado la versión española (Campos, González, \& Amor, 2002) del Vividness of Visual Imagery Questionnaire (VVIQ; Marks, 1973). El cuestionario consta de 16 ítems que se contestan, en primer lugar, con los ojos abiertos, y en segundo lugar, con los ojos cerrados. Cada ítem se puntúa en una escala de 1 a 5 puntos, el 1 indica que la imagen es "perfectamente clara y tan viva como si estuvieses viendo el objeto” y el 5 indica que la persona no tiene "ninguna imagen, tú sólo 'sabes` lo que estás pensando del objeto", por lo que las puntuaciones bajas indicaban alta viveza de imagen y viceversa.

\section{Procedimiento}

En primer lugar, se realizó la selección de las palabras a través de la obra "Normas de Imaginabilidad" de Valle Arroyo (1998). Se seleccionaron como altas en imagen, las palabras que tenían un valor de imagen mayor que 5.20, y las bajas en imagen fueron las palabras que tenían un valor de imagen inferior a 4.21. Se eliminaron todas las palabras que no fuesen sustantivos. A continuación, las palabras españolas se tradujeron al euskera por una persona nativa. Las palabras que sonasen o se escribiesen de forma similar en ambos idiomas fueron rechazadas. Posteriormente, se creó una palabra clave española para cada palabra en euskera.

La palabra clave debía de ser lo más concreta posible y que sonase los más parecido posible a la palabra en euskera. Se rechazó cualquier término cuya palabra clave creada coincidiese con alguna de las palabras de la lista inicial. Se obtuvo una primera lista de 135 sustantivos. 79 de ellos eran altos en imagen y 56 era bajos en imagen. De esta primera lista se seleccionaron, al azar, 24 palabras bajas en imagen y 24 palabras altas en imagen. A continuación se creó una palabra clave para cada palabra en euskera y una frase interactiva que uniese el significado de la palabra clave y el significado español de cada palabra en euskera. 
Las palabras en euskera se pasaron a un grupo de 48 estudiantes, de las mismas características socioeducativas que los estudiantes que se habían utilizado en la fase experimental, para que creasen las palabras clave compañeros. Se seleccionaron las palabras clave y las frases interactivas que más se repetían. A estos estudiantes se les dejó un tiempo de 50 minutos para que creasen las palabras clave y las frases interactivas.

De las 48 palabras y frases del grupo de compañeros y las de los experimentadores, se seleccionaron 28 palabras (14 palabras altas en imagen y 14 palabras bajas en imagen) procurando que la lista tuviese las siguientes condiciones: a) Que los experimentadores no hubiesen seleccionado la misma palabra clave, b) Que hubiese diferencia significativa en imaginabilidad entre las palabras altas y bajas en imagen, c) Que no hubiese diferencia significativa entre el número de sílabas de las palabras en euskera altas en imagen y las palabras en euskera bajas en imagen.

La lista de las 28 palabras fue aplicada a los participantes en el horario normal de clase. Al principio se le leyeron las instrucciones y, posteriormente, se le presentaron tres palabras como entrenamiento en un proyector de diapositivas. A continuación, se pasaron las 28 palabras que debían aprender a un ritmo de una palabra cada 15 segundos. Finalmente, se permitió a los participantes un minuto y medio para escribir, al lado de cada palabra en euskera, el significado en español de la palabra en euskera.

Durante 15 segundos se presentó cada palabra. Al grupo al que le habían generado la palabra clave los compañeros se le presentó en una diapositiva cada palabra en euskera, la palabra clave, la traducción española, y la frase interactiva que unía la palabra clave y el significado al español de la palabra en euskera. Uno de los experimentadores leía dos veces todo lo escrito en la dispositiva. En el grupo que utilizó las palabras clave generadas por los experimentadores, el proceso fue el mismo, excepto que las palabras que se les presentaron fueron las generadas por los experimentadores. Al otro grupo se le indicó que deberían aprender el significado de las palabras en euskera mediante el método de la palabra clave y que las palabras clave las debían crear en el momento del aprendizaje. Finalmente, a otro grupo se le presentaron las palabras en euskera para que aprendiesen su significado español mediante el método de repetición.

La lista de las 28 palabras fue aplicada a los participantes en el horario normal de clase. Al principio se les leyeron las instrucciones y, posteriormente, se les presentaron tres palabras como entrenamiento en un proyector de diapositivas. A continuación, se pasaron las 28 palabras que debían aprender.

Después de la prueba de recuerdo, se les pasaron a los estudiantes las pruebas de imagen: La versión española (Pérez-Fabello \& Campos, 2004) del Gordon Test of Visual Imagery Control (Richardson, 1969), y la versión española (Campos, González et al., 2002) del Vividness of Visual Imagery Questionnaire (VVIQ; Marks, 1973).

Para realizar esta investigación, se solicitaron y consiguieron los permisos pertinentes para pasar todas las pruebas. Los estudiantes participaron voluntariamente y se les garantizó la confidencialidad de los datos recogidos. La investigación se realizó siguiendo las normas éticas contenidas en la Declaración de Helsinki de 2013. Se ha conseguido, para la realización de esta investigación, la aprobación del Comité de Ética de la Universidad de Santiago de Compostela.

Para realizar esta investigación se seleccionaron, al azar, a todos los participantes. También, al azar, se han formado cuatro grupos, y también, al azar, se distribuyó a cada grupo una tarea distinta. Todas estas son características de un diseño experimental. Como criterios de inclusión se han utilizado: Que fuesen alumnos de ambos sexos (aproximadamente la mitad de cada uno), que fuesen de un centro público, que fuesen de $3^{\circ} \mathrm{y}$ $4^{\circ}$ de Enseñanza Secundaria Obligatoria (E.S.O.) (aproximadamente, la mitad de $3^{\circ}$ y la mitad de $4^{\circ}$ ), que se presentasen voluntariamente para el estudio, y que fuesen de la ciudad de A Coruña. Como criterios de exclusión se han utilizado: Que fuesen estudiantes de otros cursos de Enseñanza Secundaria Obligatoria (E.S.O.), que fuesen de colegios privados, que fuesen de colegios fuera de la ciudad de A Coruña, que, por algún motivo tuviesen algún reparo en participar en el estudio, y que no hubiesen completado todas las pruebas.

\section{Análisis de Datos}

Para analizar la consistencia interna, tanto del Gordon Test of Visual Imagery Control (Richardson, 1969), como del Vividness of Visual Imagery Questionnaire (VVIQ; Marks, 1973), se utilizó el alfa de Cronbach. 
Para analizar la influencia de la estrategia de aprendizaje (método de la palabra clave suscitada por los compañeros, por los experimentadores, por los participantes, y el método de repetición), del control de imagen (altos y bajos en control de imagen), y de la viveza de imagen (altos y bajos en viveza de imagen), en el aprendizaje de palabras, altas en imagen, se efectuó un ANOVA de 4 (estrategia de aprendizaje) x 2 (altos y bajos en control de imagen), x 2 (altos y bajos en viveza de imagen). Se efectuó un ANOVA para las palabras altas en imagen, otro para las palabras bajas en imagen, y otro para el total de palabras. Para ver la diferencia en el aprendizaje de palabras entre las estrategias de aprendizaje (compañeros, experimentadores, participantes, y repetición), tanto en las palabras altas en imagen, como bajas en imagen y total de palabras, se efectuaron Análisis de Scheffé.

\section{Resultados}

La consistencia interna ( $\alpha$ de Cronbach) de la versión española (Campos, González et al., 2002) del Vividness of Visual Imagery Questionnaire (VVIQ; Marks, 1973) fue de .91, y la consistencia interna ( $\alpha$ de Cronbach) de la versión española (Pérez-Fabello \& Campos, 2004) del Gordon Test of Visual Imagery Control (Richardson, 1969) fue de .80.

En primer lugar, se pretendía saber si la estrategia de aprendizaje (método de la palabra clave suscitada por los compañeros, por los experimentadores, por los participantes, y el método de repetición), el control de imagen (altos y bajos en habilidad de control de imagen), y la viveza de imagen (altos y bajos en habilidad para formar imágenes vivas) influía en el aprendizaje del significado de palabras en euskera, altas en imagen. Las medias y desviaciones típicas de los distintos grupos se encuentran en la Tabla 1. Los resultados del ANOVA indicaron que la estrategia de aprendizaje, $F(3,392)=4.25, p=.01, \dot{\eta}^{2}{ }_{p}=.03$, potencia $=.86$, influía en el recuerdo inmediato de palabras altas en imagen.

\section{Tabla 1}

Medias y desviaciones típicas de recuerdo del significado de palabras en euskera, en función de la estrategia de aprendizaje, del control de imagen y de la viveza de imagen

\begin{tabular}{lcccccc}
\hline & \multicolumn{7}{c}{ Recuerdo del Significado de Palabras } \\
\cline { 2 - 7 } s & \multicolumn{2}{c}{ Altas Imagen } & \multicolumn{2}{c}{ Bajas Imagen } & \multicolumn{3}{c}{ Total } \\
\cline { 2 - 7 } Grupo & $M$ & $S D$ & $M$ & $S D$ & $M$ & $S D$ \\
\hline Compañeros & 5.45 & 2.47 & 4.34 & 2.80 & 9.78 & 4.78 \\
Experimentadores & 4.19 & 2.62 & 2.57 & 2.12 & 6.76 & 4.15 \\
Participantes & 4.40 & 2.45 & 2.90 & 2.52 & 7.20 & 4.62 \\
Repetición & 4.32 & 2.22 & 2.59 & 2.17 & 6.91 & 3.85 \\
Altos Control & 4.58 & 2.48 & 3.10 & 2.52 & 7.64 & 4.52 \\
Bajos Control & 4.70 & 2.52 & 3.21 & 2.56 & 7.90 & 4.58 \\
Altos en VVIQ & 4.61 & 2.58 & 3.08 & 2.55 & 7.64 & 4.73 \\
Bajos en VVIQ & 4.62 & 2.43 & 3.19 & 2.52 & 7.80 & 4.39 \\
\hline
\end{tabular}

Nota. Altas puntuaciones en el VVIQ indican baja viveza de imagen, y viceversa.

Con la finalidad de ver entre qué grupos (método de la palabra clave suscitada por los compañeros, por los experimentadores, por los participantes, y el método de repetición) existían diferencias significativas se efectuó un Análisis de Scheffé y se encontró que el grupo compañeros recordó significativamente más palabras que el grupo participantes, que el grupo de los experimentadores, y que el grupo de repetición. No existieron diferencias significativas entre el grupo de la palabra clave generada por los experimentadores, el grupo de repetición y el grupo en el que los participantes generaron su propia palabra clave.

El control de imagen que tienen los participantes tampoco influyó en el recuerdo de palabras altas en imagen, $F(1,392)=0.39, p=.54, \dot{\eta}_{p}^{2}=.01$, potencia $=.10$, ni la viveza de imagen, $F(1,392)=0.11, p=.74$, $\dot{\eta}^{2}{ }_{p}=.01$, potencia $=.06$, de los participantes. Ninguna de las interacciones entre las variables resultó significativa: Entre la estrategia de aprendizaje y el control de imagen, $F(3,392)=0.37, p=.77, \dot{\eta}^{2} p=.01$, potencia $=.12$, entre la estrategia de aprendizaje y la viveza de imagen, $F(3,392)=0.99, p=.40, \dot{\eta}_{p}^{2}=.01$, 
potencia $=.27$, y entre la viveza de imagen y el control de imagen, $F(1,392)=0.20, p=.66, \dot{\eta}^{2}{ }_{p}=.01$, potencia $=.07$. La interacción entre las tres variables tampoco resultó significativa: La estrategia de aprendizaje, el control de imagen y la viveza de imagen, $F(3,392)=0.59, p=.62, \dot{\eta}^{2}=.01$, potencia $=.17$.

En segundo lugar, se pretendió averiguar si la estrategia de aprendizaje (método de la palabra clave suscitada por los compañeros de los participantes, por los experimentadores, por los participantes, y el método de repetición), el control de imagen (altos y bajos en habilidad de control de imagen), y la viveza de imagen (altos y bajos en habilidad de formar imágenes vivas) influía en el aprendizaje del significado de palabras en euskera, bajas en imagen. Las medias y desviaciones típicas de los distintos grupos se encuentran en la Tabla 1. Los resultados del ANOVA indicaron que la estrategia de aprendizaje influía en el recuerdo inmediato de palabras bajas en imagen, $F(3,392)=9.26, p<.001, \dot{\eta}_{p}^{2}=.07$, potencia $=1.00$.

Se efectuó un Análisis de Scheffé para ver entre qué grupos (método de la palabra clave suscitada por los compañeros, por los experimentadores, por los participantes, y el método de repetición) se encontraban las diferencias significativas. Se observó que el grupo que utilizó las palabras clave generadas por los compañeros recordó significativamente más palabras que el grupo que utilizó las palabras clave generadas por los participantes, que el grupo que utilizó las palabras clave generadas por los experimentadores, y que el grupo que utilizó el método de repetición. No existieron diferencias significativas entre el grupo de que utilizó las palabras clave de experimentadores, el grupo que utilizó el método de repetición y el grupo que utilizó sus propias palabras clave (grupo de participantes).

Ni la habilidad de control de imagen, $F(1,392)=0.61, p=.43, \dot{\eta}^{2}{ }_{p}=.01$, potencia $=.12$, ni la habilidad de los participantes de formar imágenes, $F(1,392)=0.52, p=.47, \dot{\eta}^{2}{ }_{p}=.01$, potencia $=.11$, influyó significativamente en el recuerdo inmediato del significado de palabras en euskera bajas en imagen. No resultó significativa la interacción entre la estrategia de aprendizaje y el control de imagen, $F(3,392)=0.18, p=.91$, $\dot{\eta}^{2}{ }_{p}=.01$, potencia $=.08$, entre la estrategia de aprendizaje y la viveza de imagen, $F(3,392)=1.19, p=.31, \dot{\eta}_{p}^{2}$ $=.01$, potencia $=.32$, ni entre la viveza de imagen y el control de imagen, $F(1,392)=0.36, p=.55, \dot{\eta}_{p}^{2}=.01$, potencia $=.09$. La interacción entre las tres variables (estrategia de aprendizaje, el control de imagen y la viveza de imagen) tampoco resultó significativa, $F(3,392)=0.33, p=.81, \dot{\eta}_{p}^{2}=.01$, potencia $=.11$.

Finalmente, se trató de averiguar si la estrategia de aprendizaje (método de la palabra clave suscitada por los compañeros de los participantes, por los experimentadores, por los participantes, y el método de repetición), el control de imagen (individuos altos y bajos en habilidad de control de imagen), y la viveza de imagen (individuos altos y bajos en el VVIQ) influía en el aprendizaje del significado del total de palabras del euskera. Las medias y desviaciones típicas de los distintos grupos se encuentran en la Tabla 1. Los resultados del ANOVA indicaron que la estrategia de aprendizaje, $F(3,392)=8.26, p<.001, \dot{\eta}_{p}^{2}=.06$, potencia $=.99$, influía en el recuerdo inmediato (del total) de palabras.

Para ver entre qué grupos existían diferencias significativas se efectuó un Análisis de Scheffé y encontramos que el grupo que utilizó las palabras clave generadas por sus compañeros recordó significativamente más palabras que el grupo de participantes que generó sus propias palabras clave, que el grupo que utilizó las palabras clave facilitadas por los experimentadores, y que el grupo que utilizó el método de repetición. No existieron diferencias significativas entre el grupo de las palabras clave de los experimentadores, el grupo de repetición y el grupo de los participantes que generaron sus propias palabras clave.

La capacidad de control de imágenes, $F(1,392)=0.76, p=.39, \dot{\eta}^{2}{ }_{p}=.01$, potencia $=.14$, no influyó significativamente en el recuerdo inmediato del significado de palabras en euskera, ni la viveza de imagen, $F(1,392)=0.44, p=.51, \dot{\eta}_{p}^{2}=.01$, potencia $=.10$, de los participantes. La interacción entre la estrategia de aprendizaje y el control de imagen no resultó significativa, $F(3,392)=0.29, p=.83, \dot{\eta}^{2}{ }_{p}=.01$, potencia $=.11$. Tampoco resultó significativa la interacción entre la estrategia de aprendizaje y la viveza de imagen, $F(3,392)$ $=1.08, p=.36, \dot{\eta}_{p}^{2}=.01$, potencia $=.29$, ni entre la viveza de imagen y el control de imagen, $F(1,392)=0.20$, $p=.65, \dot{\eta}_{p}^{2}=.01$, potencia $=.07$. La interacción entre la estrategia de aprendizaje, el control de imagen y la viveza de imagen, $F(3,392)=0.60, p=.61, \dot{\eta}_{p}^{2}=.01$, potencia $=.18$, tampoco resultó significativa. 


\section{Discusión}

La consistencia interna ( $\alpha$ de Cronbach) de la versión española (Pérez-Fabello \& Campos, 2004) del Gordon Test of Visual Imagery Control (Richardson, 1969) fue "buena" según la clasificación de George y Mallery (2003), y la consistencia interna ( $\alpha$ de Cronbach) de la versión española (Campos, González et al., 2002) del Vividness of Visual Imagery Questionnaire (VVIQ; Marks, 1973) fue de "excelente". Esto indica que son unas buenas medidas para investigar sobre la viveza de imagen y la habilidad de control de las imágenes mentales.

Con palabras altas en imagen, se encontró que el grupo que utilizó la palabra clave elaborada por los compañeros recordó significativamente más palabras que el grupo de participantes que elaboró las palabras clave en el momento del aprendizaje, que el grupo que utilizó la palabra clave elaborada por los experimentadores, y que el grupo que utilizó el método de repetición. Este resultado coincide con los estudios previos (Campos \& Ameijide, 2014; Campos, Amor et al., 2002, 2004a, 2004b; Campos, González et al., 2004; Campos, Pérez-Fabello et al., 2010). Este resultado indica que la mnemotecnia de la palabra clave, cuando la palabra clave es elaborada por compañeros de los participantes, es un buen método de aprendizaje del significado del euskera. Sin embargo, no hemos encontrado diferencias significativas entre el método de la palabra clave, tanto cuando la palabra clave fue elaborada por los experimentadores como por los participantes, y el método de repetición. Este resultado está en contra de un abundante número de investigaciones (Atkinson, 1975; Campos, \& Ameijide, 2014; Campos, Amor et al., 2002, 2004a, 2004b; Campos et al., 2013, 2014; Campos, González et al., 2004; Campos, Pérez-Fabello et al., 2010; Raugh \& Atkinson, 1975), que demuestran la superioridad de la mnemotecnia de la palabra clave frente a otras técnicas, en el aprendizaje de diferentes idiomas, sobre todo cuando se utilizan palabras altas en imagen que son más fáciles de imaginar que las palabras bajas en imagen (Beaton et al., 2005, Campos, Camino et al., 2010; Lawson \& Hogben, 1998; Sadoski, 2005; Shapiro \& Waters; 2005; Wei, 2015). En un estudio anterior con el euskera (González et al., 2020), tampoco se había encontrado eficacia de la mnemotecnia de la palabra clave con esos dos métodos de selección de palabras clave, utilizando listas cortas de palabras. Con palabras bajas en imagen y con el total de las palabras hemos obtenido el mismo resultado.

El control de imagen que tienen los participantes no influyó significativamente en el aprendizaje del significado de palabras en euskera, tanto con palabras altas en imagen, como palabras bajas en imagen, y en el total de palabras. Este resultado está en contra de diferentes investigaciones que analizaron esta influencia (Campos et al., 2013, 2014; Pérez-Fabello et al., 2007, 2014), si bien, existen algunas investigaciones que no encontraron el efecto positivo del control de imagen (Campos, Camino et al., 2010; Campos et al., 2013; González et al., 2020).

La viveza de imagen no influyó significativamente en el aprendizaje del significado del euskera, tanto en palabras altas en imagen, como palabras bajas en imagen, y en el total de palabras. Este resultado contradice las investigaciones que indican que la viveza de imagen de los individuos influye en el aprendizaje, sobre todo si el aprendizaje está mediado por las imágenes mentales, como la mnemotecnia de la palabra clave (Campos, Amor et al., 2002; Campos, González et al., 2004; Campos \& Pérez, 1997; Denis, 1979; Ernest, 1991; Paivio, 1971; Richardson, 1994; Wyra et al., 2007). Hay estudios que no encontraron influencia de la viveza de imagen en el aprendizaje (Campos \& Pérez, 1997; Pérez-Fabello et al., 2007).

Los estudios muy controlados, como el nuestro, tienen una gran importancia científica y pedagógica, para saber en qué circunstancias es efectiva la mnemotecnia de la palabra clave. Sin embargo, no se tiene que perder de vista que el objetivo de estas técnicas de aprendizaje es que se puedan utilizar en las clases habituales, y que los alumnos sean autónomos para utilizarlas. Por eso, el siguiente paso de estas investigaciones es conocer cómo la mnemotecnia de la palabra clave puede ser utilizada en las aulas, de forma habitual, o cómo el alumno las puede utilizar de forma autónoma (Rotjanawongchasai, 2016).

Al ser el primer estudio que se hace sobre la eficacia de la mnemotecnia de la palabra clave en el aprendizaje del euskera, con listas largas, y al haber tan pocos estudios sobre la eficacia de la mnemotecnia de la palabra clave con el euskera, el trabajo tiene limitaciones. Se estudió una edad determinada (15 años), y con un tiempo 
determinado de presentación (15 segundos para cada palabra), dos variables que influyen en el aprendizaje (Campos, González, \& Amor, 2003).

Se necesitan nuevas investigaciones que analicen la eficacia de la mnemotecnia de la palabra clave en el euskera, para analizar en qué circunstancias se debe utilizar esta estrategia. Para ello, es necesario estudiar diferentes edades de los participantes, distintos tiempos de presentación de las palabras, diferentes palabras, la longitud de las listas, y otras variables que se sabe que influyen en la eficacia de la mnemotecnia de la palabra clave, y que ya han sido estudiadas en otros idiomas (González et al., 2003).

\section{Referencias}

Atkinson, R. C. (1975). Mnemotechnics in second-language learning. American Psychologist, 30, 821-828. https://doi.org/10.1037/h0077029

Atkinson, R. C., \& Raugh, M. R. (1975). An application of the mnemonic keyword method to the acquisition of a Russian vocabulary. Journal of Experimental Psychology: Human Learning and Memory, 1, 126-133. https://doi.org/10.1037/0278-7393.1.2.126

Baleghizadeh, S., \& Ashoori, A. (2010). The effect of keyword and word-list methods on immediate vocabulary retention of EFL learners. Pakistan Journal of Social Sciences, 30, 251-261. http://pjss.bzu.edu. pk/website/journal/article/5e95531813732/page

Beaton, A., Gruneberg, M., Hyde, C., Shufflebottom, A., \& Sykes, R. (2005). Facilitation of receptive and productive foreign vocabulary learning using the keyword method: The role of image quality. Memory, 13, 458-471. https://doi.org/10.1080/09658210444000395

Campos, A., \& Ameijide, L. (2014). Mnemotecnia de la palabra clave con dibujos y juicios metamnemónicos de personas mayores. Revista Iberoamericana de Psicología y Salud, 5, 23-38.

Campos, A., Amor, A., \& González, M. A. (2002). Presentation of keywords by means of interactive drawings. The Spanish Journal of Psychology, 5, 102-109. https://doi.org/10.1017/S1138741600005874

Campos, A., Amor, A., \& González, M. A. (2004a). Drawing-assisted strategies in keyword mnemonics. Studia Psychologica, 46, 211-218. Disponible en: https://www.studiapsychologica.com/uploads/CAMPOS CAMPOS_3_vol.46_2004_pp.211-218.PDF

Campos, A., Amor, A., \& González, M. A. (2004b). The importance of the keyword-generation method in keyword mnemonics. Experimental Psychology, 51, 125-131. https://doi.org/10.1027/1618-3169.51.2.125

Campos, A., Camino, E., \& Pérez-Fabello, M. J. (2010). Aprendizaje de vocabulario con alta y baja viveza de imagen mediante la mnemotécnica de la palabra clave [Vocabulary learning with high and low imagery vividness using the mnemonic keyword method]. Revista Galego-Portuguesa de Psicoloxía e Educación, 18(2), 67-77. http://hdl.handle.net/2183/8420

Campos, A., Camino, E., \& Pérez-Fabello, M. J. (2011). Using the keyword mnemonics method among adult learners. Educational Gerontology, 37, 327-335. https://doi.org/10.1080/03601271003608886

Campos, A., González, M. A., \& Amor, A. (2002). The Spanish version of the Vividness of Visual Imagery Questionnaire: Factor structure and internal consistency reliability. Psychological Reports, 90, 503-506. https://doi.org/10.2466/pr0.2002.90.2.503

Campos, A., González, M. A., \& Amor, A. (2003). Limitations of the mnemonic-keyword method. The Journal of General Psychology, 130, 399-413. https://doi.org/10.1080/00221300309601166

Campos, A., González, M. A., \& Amor, A. (2004). Different strategies for keyword generation. Journal of Mental Imagery, $28(3 \& 4), 51-58$.

Campos, A., \& Pérez, M.J. (1997). Mnemonic images and associated pair recall. Journal of Mental Imagery, $21,73-82$.

Campos, A., Pérez-Fabello, M. J., \& Camino, E. (2010). Eficacia de la mnemotecnia de la palabra clave en personas adultas. Psicothema, 22, 752-757. http://www.psicothema.com/psicothema.asp?id=3797

Campos, A., Rodríguez-Pinal, \& Pérez-Fabello (2013). Aprendizaje del idioma gallego mediante la mnemotecnia de la palabra clave, en personas bilingüies, altas y bajas en control de imagen. Revista de Investigación en Educación, 11(2), 50-59. http://reined.webs.uvigo.es/index.php/reined/article/view/173 
Campos, A., Rodríguez-Pinal, \& Pérez-Fabello (2014). Receptive and productive recall with the keyword mnemonics in bilingual students. Current Psychology, 33, 64-72. https://doi.org/10.1007/s12144-013-9197-y Davoudi, M., \& Yousefi, D. (2016). The effect of keyword method on vocabulary retention of senior high school EFL learners in Iran. Journal of Education and Practice, 7(11), 106-113.

Denis, M. (1979). Les images mentales. Paris: Puf.

Ernest, C. H. (1991). Ability differences and prose learning. Intelligence, 15, 455-477. https://doi.org/10.1016/ 0160-2896(91)90007-Z

George, D., \& Mallery, P. (2003). SPSS for Windows steps by step: A simple guide and reference. 11.0 update. Boston: Allyn \& Bacon.

González, M. A., Amor, A., \& Campos, A. (2003). La mnemotecnia de la palabra clave. A Coruña: Servicio de Publicaciones de la Universidad de A Coruña.

González, M. A., Goñi-Artola, A., \& Campos, A. (2019). The effect of the concordance of peer-generated keywords on language acquisition. North American Journal of Psychology, 21, 423-440.

González, M. A., Goñi-Artola, A., \& Campos, A. (2020). Eficacia de la mnemotecnia de la palabra clave y la viveza de imagen en el aprendizaje del euskera. Manuscrito enviado para publicación.

Hall, J. W., Wilson, K. P., \& Patterson, R. J. (1981). Mnemotechnics: Some limitations of the mnemonic keyword method for the study of foreign language vocabulary. Journal of Educational Psychology, 73, 345357. https://doi.org/10.1037/0022-0663.73.3.345

Hogben, D., \& Lawson, M. J. (1994). Keyword and multiple elaboration strategies for vocabulary acquisition in foreign language learning. Contemporary Educational Psychology, 19, 367-376. https://doi.org/10.1006/ ceps.1994.1027

Lawson, M. J., \& Hogben, D. (1998). Learning and recall of foreign-language vocabulary: Effects of a keyword strategy for immediate and delayed recall. Learning and Instruction, 8, 179-194. https://doi.org/10.1016/ S0959-4752(97)00016-9

Levin, J. R., McCormick, C. B., Miller, G. E., Berry, J. K., \& Pressley, M. (1982). Mnemonic versus nonmnemonic vocabulary-learning strategies for children. American Educational Research Journal, 19, 121-136. https://doi.org/10.3102/00028312019001121

Levin, J. R., Pressley, M., McCormick, C. B., Miller, G. E., \& Shriberg, L. K. (1979). Assessing the classroom potential of the keyword method. Journal of Educational Psychology, 71, 583-594. https://doi.org/10.1037/ 0022-0663.71.5.583

Marks, D. F. (1973). Visual imagery differences in the recall of pictures. British Journal of Psychology, 64, 17-24. https://doi.org/10.1111/j.2044-8295.1973.tb01322.x

Merry, R. (1980). The keyword method and children's vocabulary learning in the classroom. British Journal of Educational Psychology, 50, 123-136. https://doi.org/10.1111/j.2044-8279.1980.tb02438.x

Paivio, A. (1971). Imagery and verbal processes. New York: Holt, Rinehart and Winston.

Pérez-Fabello, M. J., \& Campos, A. (2004). Factor structure and internal consistency of the Spanish version of the Gordon Test of Visual Imagery Control. Psychological Reports, 94, 761-766. https://doi.org/10.2466/ pr0.94.3.761-766

Pérez-Fabello, M. J., \& Campos, A., \& Gómez-Juncal, R. (2007). Visual imaging capacity and imagery control in fine arts students. Perceptual and Motor Skills, 104, 815-822. https://doi.org/10.2466/pms.104.3.815-822

Pérez-Fabello, M. J., \& Campos, A., \& Meana, J. C. (2014). Vividness and control of mental imagery and the component of in-depth drawing. Creativity Research Journal, 26, 244-247. https://doi.org/10.1080/ 10400419.2014.901097

Pressley, M., Samuel, J., Hershey, M. M., Bishop, S. L., \& Dickinson, D. (1981). Use of a mnemonic technique to teach young children foreign language vocabulary. Contemporary Educational Psychology, 6, 110-116. https://doi.org/10.1016/0361-476X(81)90039-4

Raugh, M. R., \& Atkinson, R. C. (1975). A mnemonic method for the learning of a second language vocabulary. Journal of Educational Psychology, 67, 1-16. https://doi.org/10.1037/h0078665 
Richardson, A. (1969). Mental imagery. Nueva York: Springer.

Richardson, A. (1994). Individual differences in imaging. Amityville, NY: Baywood.

Rotjanawongchasai, S. (2016). How teachers can make the keyword method more challenging for students. The Asian EFL Journal Professional Teaching Articles, 96, 60-82.

Sadoski, M. (2005). A dual coding view of vocabulary learning. Reading \& Writing Quarterly, 2, 221-238. https://doi.org/10.1080/10573560590949359

Shapiro, A. M., \& Waters, D. L. (2005). An investigation of the cognitive processes underlying the keyword method of foreign vocabulary learning. Language Teaching Research, 9, 129-146. https://doi.org/10.1191/ 13621688051r151oa

Troutt-Ervin, E. (1990). Application of keyword mnemonics to learning terminology in the college classroom. The Journal of Experimental Education, 59, 31-41. https://doi.org/10.1080/00220973.1990.10806549

Tulving, E. (2002). Episodic memory: From mind to brain. Annual Review of Psychology, 53, 1-25. https://doi.org/10.1146/annurev.psych.53.100901.135114

Valle Arrollo, F. (1998). Normas de imaginabilidad. Oviedo: Servicio de Publicaciones de la Universidad de Oviedo.

Wei, Z. (2015). Does teaching mnemonics for vocabulary learning make a difference? Putting the keyword method and the word part technique to the test. Language Teaching Research, 19, 43-69. https://doi.org/ $10.1177 / 1362168814541734$

Wyra, M., Lawson, M. J., \& Hungi, N. (2007). The mnemonic keyword method: The effects of bidirectional retrieval training and of ability to image on foreign language vocabulary recall. Learning and Instruction, 17, 360-371. https://doi.org/10.1016/j.learninstruc.2007.02.008

Fecha de recepción: 4 de abril de 2020.

Fecha de revisión: 4 de mayo de 2020.

Fecha de aceptación: 12 de mayo de 2020.

Fecha de publicación: 1 de julio de 2020. 